\title{
Heparanase mRNA and Protein Expression Correlates with Clinicopathologic Features of Gastric Cancer Patients: a Meta- analysis
}

\author{
Hai-Long $\mathrm{Li}^{1,2 \&}$, Jing $\mathrm{Gu}^{2,3 \&}$, Jian-Jun $\mathrm{Wu}^{4}$, Chun-Lin $\mathrm{Ma}^{2,3}$, Ya-Li Yang1, Hu- \\ Ping Wang ${ }^{2,3}$, Jing Wang ${ }^{1}$, Yong Wang ${ }^{1}$, Che Chen ${ }^{1}$, Hong-Yan Wu ${ }^{2,3 *}$
}

\begin{abstract}
Background: Heparanase is believed to be involved in gastric carcinogenesis. However, the clinicopathologic features of gastric cancer with high heparanase expression remain unclear. Aim : The purpose of this study was to comprehensively and quantitatively summarize available evidence for the use of heparanase mRNA and protein expression to evaluate the clinicopathological associations in gastric cancer in Asian patients by meta-analysis. Materials and Methods: Relevant articles listed in MEDLINE, CNKI and the Cochrane Library databases up to MARCH 2015 were searched by use of several keywords in electronic databases. A meta-analysis was performed to clarify the impact of heparanase mRNA and protein on clinicopathological parameters in gastric cancer. Combined ORs with 95\% CIs were calculated by Revman 5.0, and publication bias testing was performed by stata12.0. Results: A total of 27 studies which included 3,891 gastric cancer patients were combined in the final analysis. When stratifying the studies by the pathological variables of heparanase mRNA expression, the depth of invasion (633 patients) $(O R=4.96 ; 95 \% \mathrm{CI}=2.38-1.37 ; P<0.0001)$, lymph node metastasis (639 patients) $(\mathrm{OR}=6.22$; 95\% $\mathrm{CI}=2.70-14.34, P<0.0001)$, and lymph node metastasis $(383$ patients $)(\mathrm{OR}=6.85 ; 95 \% \mathrm{CI}=2.04-23.04 ; P=0.002)$ were all significant. When stratifying the studies by the pathological variables of heparanase protein expression, this was the case for depth of invasion (1250 patients) $(\mathrm{OR}=2.76 ; 95 \% \mathrm{CI}=1.52-5.03 ; P=0.0009)$, lymph node metastasis $(1178$ patients $)(\mathrm{OR}=4.79 ; 95 \% \mathrm{CI}=3.37-6.80, P<0.00001)$, tumor size $(727$ patients $)(\mathrm{OR}=2.06 ; 95 \%$ CI $=1.31-3.23 ; P=0.002)(O R=2.61 ; 95 \% C I=2.09-3.27 ; P=0.000)$, and TNM stage $(1233$ patients $)(O R=6.85 ; 95 \%$ $\mathrm{CI}=2.04-23.04 ; \boldsymbol{P}=\mathbf{0 . 0 0 2}$ ). Egger's tests suggested publication bias for depth of invasion, lymph node metastasis, lymph node metastasis and tumor size of heparanase mRNA and protein expression. Conclusions: This metaanalysis suggests that higher heparanase expression in gastric cancer is associated with clinicopathologic features of depth of invasion, lymph node metastasis and TNM stage at mRNA and protein levels, and of tumor size only at the protein level. Egger's tests suggested publication bias for these clinicopathologic features of heparanase mRNA and protein expression, and which may be caused by shortage of relevant studies. As a result, although abundant reports showed heparanase may be associated with clinicopathologic features in gastric cancer, this meta-analysis indicates that more strict studies were needed to evaluate its clinicopathologic significance.
\end{abstract}

Keywords: Gastric cancer - heparanase - clinicopathologic features - meta-analysis

Asian Pac J Cancer Prev, 16 (18), 8653-8658

\section{Introduction}

Heparanase is an endo-B-Dglucuronidase that specifically cleaves carbohydrate chains of heparan sulfate proteoglycans (HSPG) (Nadir and Brenner, 2014), the main polysaccharide constituent of the extracellular matrix and basement membrane. Overexpression of heparanase has been found in numerous tumor types and associated with poor prognosis, and because of its peculiar action in tumorgenesis of cancer, heparanase has become a ideal cancer biomarker with many indications and a potential therapeutic target with multiple actions of anti-tumor, anti-angiogenic and anti-inflammatory (Masola et al., 2014; Pisano et al., 2014). As for gastrointestinal cancer, heparanase has been thought to play an important role in the process of invasion and metastasis (Zheng et al., 2009; Wu et al., 2010; Zheng et al., 2010), and even prognosis (Naomoto et al., 2005). However, reports about the clinicopathologic features of high heparanase expression in gastric cancer was not fully same, and conflicting and inconclusive, and even some features of high heparanase expression were missing (Chen et al.,

${ }^{1}$ School of Medicine Technology, ${ }^{2}$ Key Laboratory of Traditional Chinese Herbs and Prescription Innovation and Transformation of Gansu Province, ${ }^{3}$ School of Basic Medical Sciences, ${ }^{4}$ School of Public Health Institute, Gansu University of Traditional Chinese Medicine, Lanzhou, China ${ }^{\star}$ Equal contributors *For correspondence: wu.hy@126.com 
2004a; Chen et al., 2004b; Zhang et al., 2012; Zheng et al., 2012). Meta-analysis is becoming more and more popular in evaluating tumor biomarkers about its expressions in cancer with clinicopathological factors (Liu et al., 2013; Han et al., 2014; Liu et al., 2014). In view of the expression of heparanase mRNA and protein in gastric cancer, it is valuable to extract important information of clinicopathological factors and to evaluate the correlation between its expressions and clinicopathological factors by meta-analysis.

\section{Materials and Methods}

Search Strategy and Study Selection Relevant articles studying the relationship between heparanase mRNA and protein and clinicopathologic features of gastric cancer patients published up to July, 2013, were retrieved by online search in PubMed and China National Knowledge Infrastructure. We used the keywords: ("heparanase" or "HPSE") and ("gastric cancer" or "gastric neoplasms" or "gastric carcinoma" or "stomach cancer" or "stomach neoplasms" or "stomach carcinoma") and ("prognostic" or "prognosis" or "survival" or "survive"). All included studies were required to be written in English or Chinese. References of the original studies were also checked, to ensure all eligible studies could be included, heparanase expression of gastric cancer tissues comparing with adajacent non cancerous tissues were statistically calculated by $\mathrm{x}^{2}$ test. Accordingly, The excluding criterion were as follows: review articles, simple commentaries, case reports, or unpublished reports, heparanase expression of gastric cancer tissues comparing with adajacent non cancerous tissues were statistically calculated by student's test.

\section{Data extraction and quality assessment}

Two authors ( $\mathrm{Li}$ and $\mathrm{Gu}$ ) independently extracted information from eligible articles, including year of publication, name of the first author, country, number of patients, years of follow up, TNM stage, patients characteristics, experimental method, cut off value, percentage of heparanase positive expression, analytical method, HR, and $95 \%$ CI from the included articles. We conducted a quality assessment for each eligible study by using reporting recommendations for tumor marker prognostic studies (REMARK). which have been used in previous meta-analysis (McShane et al., 2006).

\section{Statistical analysis}

Results were expressed with risk ratio (RR) for dichotomous data, and $95 \%$ confidence intervals (CI) were counted. $P<0.05$ was required for the overall RR to be statistically significant. In this meta-analysis, HR and $95 \%$ CI were used to calculate the overall effect estimate. Heterogeneity was assessed by the Chi-squared test and $p$ value in our meta-analysis. Using $\mathrm{I}^{2}$ value to evaluate the heterogeneity, fixed-effect model was used if there was $I^{2}=0-50 \%$, which means no significant heterogeneity. Otherwise, the random-effects model was applied. Funnel plots and Egger' linear regression test were used to assess evidence for publication bias. All $p$ values were two-side, being statistically significant when $p$ value less than 0.05 . All statistical tests for this meta-analysis were performed using REVMAN5.0 software, and publication bias testing was also performed by stata12.0 software.

\section{Results}

\section{Eligible study characteristics}

A total of 27 studies from including 10 studies about heparanase mRNA expressions and 17 studies heparanase protein expressions (Table 1) were found to meet the criteria for this analysis after the article titles, abstracts and main text were read to identify case reports and clinical outcomes. The total number of patients of heparanase mRNA expressions was 685, including 445 cases heparanase + gastric cancer and a 240 controls; the total number of patients of heparanase mRNA expressions was 1299 ,including 820 cases heparanase + gastric cancer and a 479 controls. RT-PCR and In Situ Hybridization was a primary method used to evaluate the expressions of heparanase mRNA in gastric cancer specimens, while immunohistochemistry (IHC) was an essential method used to evaluate the expressions of heparanase protein in gastric cancer specimens. Studies were carried out in Japan, China. Table 1 presents the study characteristics for the included trials.

Meta-analysis results Correlation of heparanase mRNA expression with clinicopathological features

When stratifying for the different variables by the depth of invasion of gastric cancer, statistical significance was observed. Patients with T3 and T4 gastric cancer had a much higher heparanase expression in 9 studies (633 patients) $(\mathrm{OR}=4.96 ; 95 \% \mathrm{CI}=2.38-1.37 ; P<0.0001)$ than those with $\mathrm{T} 1$ and $\mathrm{T} 2$ gastric cancer (Figure 1, Table 2). When stratifying for lymph node status of gastric cancer, statistically significant results also illustrated that heparanase expression was associated with lymph node metastasis in 9 studies (639 patients) ( $O R=6.22$; 95\% CI=2.70-14.34, $P<0.0001$ ) (Figure 2, Table 2). When further stratifying for TNM stage, heparanase expression of patients with stages III and IV gastric cancer was much higher than those with stage I and II gastric cancer in 6 studies (383 patients) $(\mathrm{OR}=6.85 ; 95 \%$ $\mathrm{CI}=2.04-23.04 ; P=0.002$ ) (Figure 3, Table 2). However, there was no significant relationship between heparanase overexpression and gender, histologic differentiation, distant metastasis, vascular invasion, borrmann type, lymphatic invasion, tumor size of patients with gastric cancer.

\section{Correlation of heparanase protein expression with clinicopathological features}

When stratifying for the different variables by the depth of invasion of gastric cancer, statistical significance was observed. Patients with T3 and T4 gastric cancer had a much higher heparanase expression in 15 studies (1250 patients $)(\mathrm{OR}=2.76 ; 95 \% \mathrm{CI}=1.52-5.03 ; P=0.0009)$ than those with T1 and T2 gastric cancer (Figure 4, Table 3 ). When stratifying for lymph node status of gastric cancer, statistically significant results also showed that 
Table 1. TheCorrelation of Heparanase mRNA Expression with Clinicopathological Features

\begin{tabular}{|c|c|c|c|c|c|}
\hline References of studies & $\begin{array}{l}\text { Nation } \\
\text { Positive }\end{array}$ & $\begin{array}{l}\text { Language } \\
\text { Negative }\end{array}$ & Heparanase & Heparanase & Method \\
\hline (Inoue et al., 2001) & Japan & English & 42 & 29 & RT-PCR \\
\hline (Tang et al., 2002) & Japan & English & 96 & 20 & In Situ Hybridization \\
\hline (Takaoka et al., 2003) & Japan & English & 35 & 9 & IHC \\
\hline (REN et al., 2003) & China & Chinese & 25 & 27 & In situ hybridization \\
\hline (Chen et al., 2004a) & China & English & 29 & 14 & $\mathrm{IHC}$ \\
\hline (Liu et al., 2004) & China & Chinese & 34 & 26 & $\mathrm{IHC}$ \\
\hline (Zheng et al., 2004) & China & Chinese & 29 & 21 & RT-PCR \\
\hline (Wang et al., 2005) & China & English & 14 & 16 & RT-PCR \\
\hline (Sun et al., 2005) & China & Chinese & 60 & 37 & IHC \\
\hline (Cai et al., 2004) & China & Chinese & 35 & 12 & RT-PCR \\
\hline (Ru et al., 2006 & China & Chinese & 67 & 51 & In situ hybridization \\
\hline (QIN et al., 2007) & China & Chinese & 39 & 16 & $\mathrm{IHC}$ \\
\hline (Ma et al., 2007) & China & Chinese & 98 & 40 & IHC \\
\hline (Liu et al., 2007) & China & Chinese & 48 & 2 & IHC \\
\hline (Huang et al., 2008) & China & Chinese & 39 & 16 & $\mathrm{IHC}$ \\
\hline (Wang et al., 2008) & China & Chinese & 41 & 15 & RT-PCR \\
\hline (Liang et al., 2009) & China & Chinese & 49 & 31 & $\mathrm{IHC}$ \\
\hline (XI et al., 2009) & China & Chinese & 46 & 44 & In situ hybridization \\
\hline (Cheng et al., 2009) & China & Chinese & 39 & 26 & $\mathrm{IHC}$ \\
\hline (Su et al., 2009) & China & Chinese & 39 & 24 & $\mathrm{IHC}$ \\
\hline (Zhang et al., 2009) & China & Chinese & 49 & 31 & $\mathrm{IHC}$ \\
\hline (Qi et al., 2010) & China & Chinese & 50 & 5 & RT-PCR \\
\hline (Cheng et al., 2010) & China & English & 52 & 50 & $\mathrm{IHC}$ \\
\hline (Jiang et al., 2011) & China & Chinese & 40 & 20 & IHC \\
\hline (Zhang et al., 2012) & China & English & 52 & 80 & IHC \\
\hline (Li tao, et al., 2012) & China & Chinese & 51 & 29 & IHC \\
\hline (Zhang et al., 2013) & China & English & 67 & 28 & IHC \\
\hline
\end{tabular}

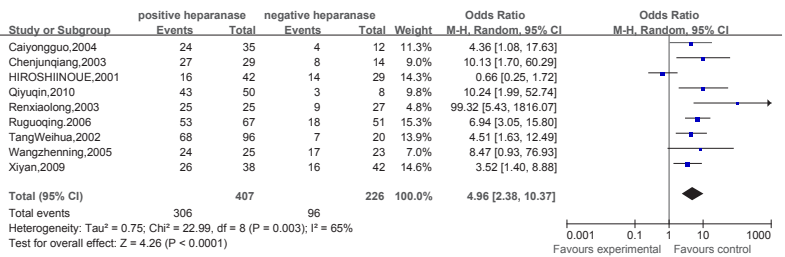

Figure 1. Forest Plot Analysis Showed that Patients with T3 and T4 Gastric Cancer had a Much Higher Heparanase Expression in 9 Sudies (633 patients) (OR $=4.96 ; 95 \% \mathrm{CI}=2.38-1.37 ; P<0.0001)$ than those with T1 and T2 Gastric Cancer

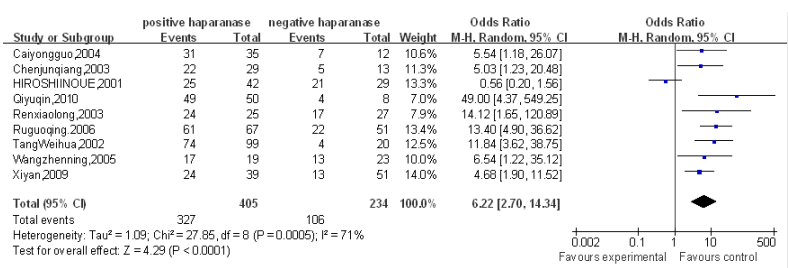

Figure 2. Forest Plot Analysis Showed that Heparanase Expression was Associated with Lymph Node Metastasis in 9 Studies (639 Patients) $(\mathrm{OR}=6.22 ; 95 \%$ CI=2.70-14.34, $P<0.0001)$

heparanase expression was associated with lymph node metastasis in 15 studies (1178 patients) $(\mathrm{OR}=4.79 ; 95 \%$ $\mathrm{CI}=3.37-6.80, P<0.00001)$ (Figure 5, Table 3). When further stratifying for TNM stage, heparanase expression of patients with stages III and IV gastric canvcer was much higher than those with stage I and II gastric cancer in 15 studies (1233 patients) $(\mathrm{OR}=4.66$; $95 \% \mathrm{CI}=2.50$ $8.68 ; P<0.00001)$ (Figure 6, Table 3). When stratifying

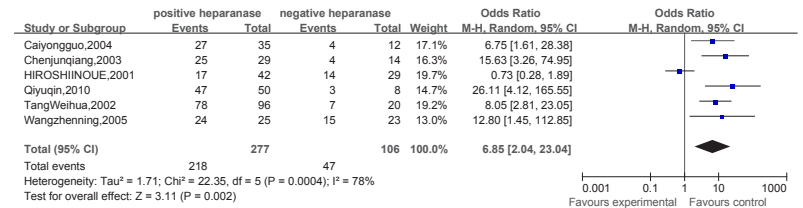

Figure 3. Forest Plot Analysis Showed that Heparanase Expression of Patients with stages III and IV Gastric Cancer was Much Higher than those with Sage I and II Gastric Cancer in 6 Studies (383 Patients) (OR = 6.85; 95\% CI = 2.04-23.04; $P=0.002$ )

for tumor size, heparanase expression of patients with tumor diameter larger than $5 \mathrm{~cm}$ was much higher than those with tumor diameter less than $5 \mathrm{~cm}$ in 8 studies $(727$ patients) $(\mathrm{OR}=2.06 ; 95 \% \mathrm{CI}=1.31-3.23 ; P=0.002)$ (Figure 7, Table 3). However, there was no significant relationship between heparanase overexpression and gender, histologic differentiation, distant metastasis, vascular invasion, borrmann type, lymphatic invasion, tumor size of patients with gastric cancer.

\section{Publication bias}

In order to clarify the clinicopathologic features of depth of invasion, lymph node metastasis, TNM stage and tumor size in gastric cancer. Egger's tests were carried out to analysis publication bias for these clinicopathologic features of heparanase mRNA and protein expression. Results indicated that publication bias were found in depth of invasion, lymph node metastasis, TNM stage and tumor size with heparanase overexpression at mRNA level, and publication bias were found in depth of 
Table 2. The Correlation of Heparanase mRNA Expression with Clinicopathological Features

\begin{tabular}{lcccrcc}
\hline Clinicopathologic & $\begin{array}{c}\text { Number } \\
\text { Features }\end{array}$ & $\begin{array}{c}\text { Number } \\
\text { of patientss }\end{array}$ & $\begin{array}{c}\text { HR } \\
(95 \% \mathrm{CI})\end{array}$ & \multicolumn{3}{c}{ Heterogeneity } \\
\cline { 4 - 7 } Gender & 335 & 5 & $0.86[0.50,1.47]$ & 2.77 & $0 \%$ & 0.6 \\
Depth of invasion & 633 & 9 & $4.96[2.38,10.37]$ & 22.99 & $65 \%$ & 0.003 \\
Differentiation of cell & 373 & 6 & $1.02[0.55,1.88]$ & 8.37 & $40 \%$ & 0.14 \\
Lymph node metastasis & 637 & 9 & $6.22[2.70,14.34]$ & 27.85 & $71 \%$ & 0.0005 \\
Distant metastasis & 494 & 7 & $4.38[0.88,21.76]$ & 36.44 & $84 \%$ & $<0.00001$ \\
TNM stage & 383 & 6 & $6.85[2.04,23.04]$ & 22.35 & $78 \%$ & 0.0004 \\
Tumor size & 208 & 3 & $2.85[0.74,10.98]$ & 5.35 & $63 \%$ & 0.07 \\
Vascular invasion & 305 & 3 & $3.03[0.19,48.35]$ & 24.88 & $92 \%$ & $<0.00001$ \\
\hline
\end{tabular}

Table 3. The Correlation of Heparanase Protein Expression with Clinicopathological Features

\begin{tabular}{lcccccc}
\hline $\begin{array}{l}\text { Clinicopathologic } \\
\text { Features }\end{array}$ & $\begin{array}{c}\text { Number } \\
\text { of patientss }\end{array}$ & $\begin{array}{c}\text { Number } \\
\text { of studies }\end{array}$ & $\begin{array}{c}\text { HR } \\
(95 \% \mathrm{CI})\end{array}$ & \multicolumn{3}{c}{ Heterogeneity } \\
\cline { 3 - 6 } Gender & 1063 & 12 & $1.12[0.85,1.46]$ & 8.17 & $0 \%$ & 0.7 \\
Depth of invasion & 1250 & 15 & $2.76[1.52,5.03]$ & 67.08 & $79 \%$ & $<0.00001$ \\
Differentiation of cell & 1245 & 15 & $1.21[0.78,1.87]$ & 40.02 & $65 \%$ & 0.0003 \\
Lymph node metastasis & 1178 & 16 & $4.55[3.54,5.85]$ & 24.53 & $43 \%$ & 0.05 \\
Distant metastasis & 621 & 7 & $4.03[0.74,21.81]$ & 36.02 & $83 \%$ & $<0.00001$ \\
TNM stage & 1233 & 15 & $4.66[2.50,8.68]$ & 68.2 & $79 \%$ & $<0.00001$ \\
Tumor size & 727 & 8 & $2.06[1.31,3.23]$ & 12.83, & $45 \%$ & 0.002 \\
\hline
\end{tabular}

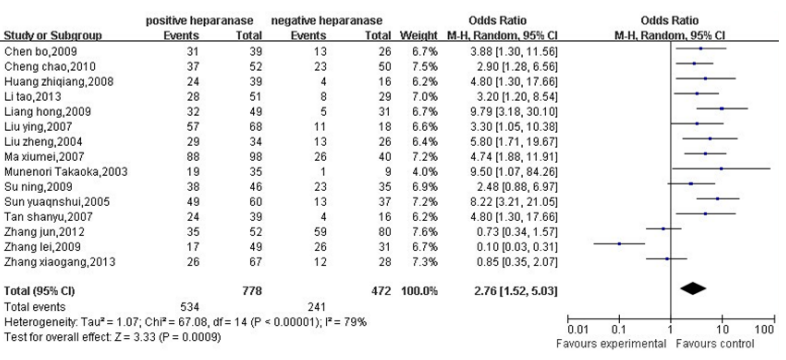

Figure 4. Forest Plot Analysis Showed that Patients with T3 and T4 Gastric Cancer had a Much Higher Heparanase Expression in 15 Studies (1250 Patients) $(\mathrm{OR}=2.76 ; 95 \% \mathrm{CI}=1.52-5.03 ; P=0.0009)$ than those with $\mathrm{T} 1$ and $\mathrm{T} 2$ Gastric Cancer

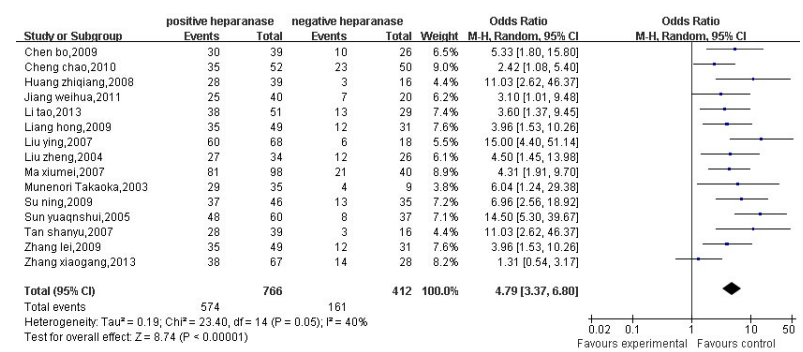

Figure 5. Forest Plot Analysis Showed that Heparanase Expression was Associated with Lymph Node Metastasis in 15 Studies (1178 Patients) $(O R=4.79$; 95\% CI=3.37-6.80, $P<0.00001)$

invasion, lymph node metastasis, TNM stage and tumor size with heparanase overexpression at protein level. The reasons may be caused by shortage of relevant studies with less than 10 studies included in mRNA heparanase overexpression, and less than 20 studies included in heparanase protein overexpression.

\section{Discussion}

Abundant clinical studies showed that overexpression of heparanase is correlated with clinicopathological

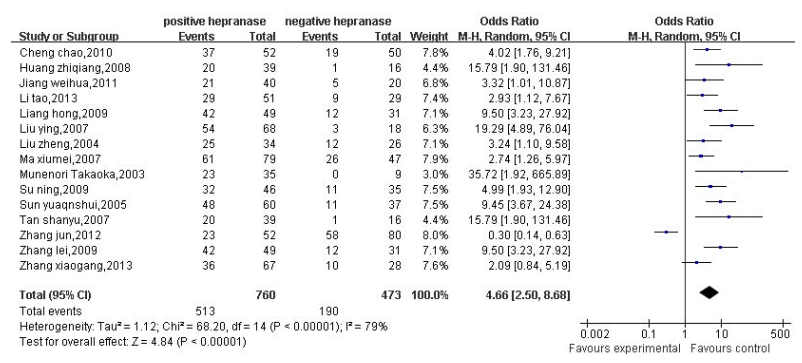

Figure 6. Forest Plot Analysis Showed that Heparanase Expression of Patients with Stages III and IV Gastric Cancer was Much Higher than those with Stage I and II Gastric Cancer in 15 Studies (1233 Patients) $(O R=4.66$; 95\% CI=2.50-8.68; $P<0.00001)$

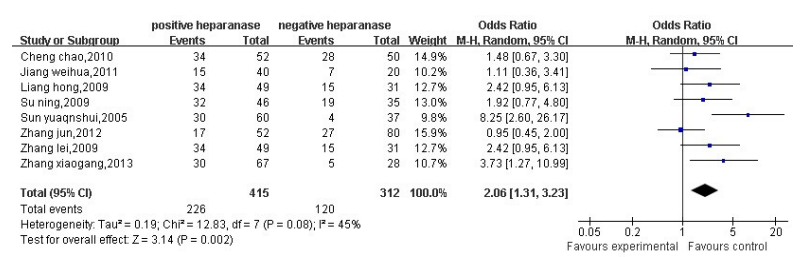

Figure 7. Forest plot analysis showed that heparanase expression of patients with tumor diameter larger than $5 \mathrm{~cm}$ was much higher than those with tumor diameter less than $5 \mathrm{~cm}$ in 8 studies (727 patients) $(O R=2.06$; 95\% CI $=1.31-3.23 ; P=0.002$ )

features, in particular, with metastasis and poor prognosis of a series of cancers including gastric cancer. All clinical data and knockdown experiment (Inoue et al., 2001; REN et al., 2003; Takaoka et al., 2003; Chen et al., 2004b; Wang et al., 2005; XI et al., 2009; Zheng et al., 2009; Zheng et al., 2010 ) in gastric cancer cells in vitro proved that high expression of heparanase to be a potential tumor biomarker and a strong predictor of poor survival. In recent years, meta-analysis has been used to screen and evaluate tumor biomarker and their clinical significances. Plenty of clinical reports suggested that heparanase could be a promising tumor biomarker for metastasis and prognosis 
of gastric cancer, and whereas no systematic review of clinical studies about high expression of heparanase in gastric cancer was carried out until now. Hence, it is necessary to review the clinicopathological features of high expression of heparanase systematically by metaanalysis to discover potential tumor biomarkers.

In this meta-analysis, we found high heparanase mRNA expression, as detected by RT-PCR and In Situ Hybridization, and high heparanase protein expression, as detected by immunohistochemistry was confirmed in patients with gastric cancer according to the evidencebased medicine. The pooled statistical data showed that heparanase mRNA and protein, when stratifying for baseline characteristics of patients, including sex, age, tumor size, histo-differentiation, depth of invasion, lymph node status, distant metastasis, vascular invasion, borrmann type, and TNM stage, our present meta-analysis indicated that over expression of heparanase mRNA was associated with depth of invasion, lymph node metastasis and TNM stage, and over expression of heparanase protein was significantly associated with the depth of invasion, lymph node metastasis, TNM stage and tumor size. However, Egger's tests showed that the clinicopathologic features of depth of invasion, lymph node metastasis, and TNM stage and tumor size in gastric cancer showed publication bias for these clinicopathologic features of heparanase mRNA and protein expression. Certainly, these published articles included in this meta-analysis are less than 20, which will lead to bias if egger's tests were adopted to test publication bias. So more serious studies were needed to review clinicopathologic significances of overexpression of heparanase mRNA and protein. Plenty of studies of heparanase mRNA and protein expression reported to be associated with clinicopathological features in different types of cancer including gastric cancer, lung cancer, acute myeloid leukemia, breast, prostate, hepatocellular, pancreatic, colon cancer.

In conclusion, this meta-analysis results of clinicopathologic features showed that overexpression of heparanase mRNA and protein are correlated with depth of invasion, lymph node metastasis, tumor size and TNM stage. Heparanase still might serve as an efficient marker for clinicopathologic features indicator, and could be a new molecular target in gastric cancer therapy. In addition, because of publication bias for these clinicopathologic significances exist suggested by Egger's tests in these published articles, if more serious and eligible studies were included for meta-analysis, it will promote the understanding of clinicopathologic significances of heparanase in gastric cancer.

\section{Acknowledgements}

This study was supported by Natural Science Fund Program of Gansu Province [number: 1310RJZA099].

\section{References}

Cai YG, Fang DC, Yang SM, et al (2004). [Increased expression of heparanase mRNA is correlated with metastasis of gastric carcinoma and is probably correlated with c-met protein]. Zhonghua Yi Xue Za Zhi, 84, 974-8.

Chen JQ, Zhan WH, He YL, et al (2004a). Expression of heparanase gene, CD44v6, MMP-7 and nm23 protein and their relationship with the invasion and metastasis of gastric carcinomas. World J Gastroenterol, 10, 776-82.

Chen JQ, Zhan WH, He YL, et al (2004b). [Relationship between heparanase mRNA expression in human gastric cancer and its clinicopathological features]. Zhonghua Zhong Liu Za Zhi, 26, 609-11.

Cheng C, Lu X, Wang G, et al (2010). Expression of SATB1 and heparanase in gastric cancer and its relationship to clinicopathologic features. APMIS, 118, 855-63.

Han Y, Xue XF, Shen HG, et al (2014). Prognostic significance of Beclin-1 expression in colorectal cancer: a meta-analysis. Asian Pac J Cancer Prev, 15, 4583-7.

Inoue H, Mimori K, Utsunomiya T, et al (2001). Heparanase expression in clinical digestive malignancies. Oncol Rep, 8, 539-42.

Liu WJ, Tan XH, Guo BP, et al (2013). Associations between RASSF1A promoter methylation and NSCLC: a metaanalysis of published data. Asian Pac J Cancer Prev, 14, 3719-24.

Liu Y, Lv DL, Duan JJ, et al (2014). ALDH1A1 expression correlates with clinicopathologic features and poor prognosis of breast cancer patients: a systematic review and metaanalysis. BMC Cancer, 14, 444.

Masola V, Secchi MF, Gambaro G, et al (2014). Heparanase as a target in cancer therapy. Curr Cancer Drug Targets, 14, 286-93.

McShane LM, Altman DG, Sauerbrei W, et al (2006). REporting recommendations for tumor MARKer prognostic studies (REMARK). Breast Cancer Res Treat, 100, 229-35.

Nadir Y, Brenner B (2014). Heparanase multiple effects in cancer. Thromb Res, 133 Suppl 2, 90-4.

Naomoto Y, Takaoka M, Okawa T, et al (2005). The role of heparanase in gastrointestinal cancer (Review). Oncol Rep, 14, 3-8.

Pisano C, Vlodavsky I, Ilan N, et al (2014). The potential of heparanase as a therapeutic target in cancer. Biochem Pharmacol, 89, 12-9.

QIN S-y, JIANG H-x, LI X (2007). Significance of bFGF, FGFR-2 and Hpa expression in gastric precancerous lesions and gastric carcinoma tissues. Chinese J Practical Internal Med, 17, 025.

REN X-1, ZHU R-z, WANG L-j (2003). Clinicopathological significance of heparanase mRNA expression in gastric carcinoma. Chinese J Exp Surg, 9, 006.

Takaoka M, Naomoto Y, Ohkawa T, et al (2003). Heparanase expression correlates with invasion and poor prognosis in gastric cancers. Lab Invest, 83, 613-22.

Tang W, Nakamura Y, Tsujimoto M, et al (2002). Heparanase: a key enzyme in invasion and metastasis of gastric carcinoma. Mod Pathol, 15, 593-8.

Wang Z, Xu H, Jiang L, et al (2005). Positive association of heparanase expression with tumor invasion and lymphatic metastasis in gastric carcinoma. Mod Pathol, 18, 205-11.

Wu BW, Li DF, Ke ZF, et al (2010). Expression characteristics of heparanase in colon carcinoma and its close relationship with cyclooxygenase-2 and angiogenesis. Hepatogastroenterol, 57, 1510-4.

Xi Y, Zhang Y, Qi YJ, et al (2009). Expression of heparanase and CD105 in stomach neoplasm and its correlation. J Henan University, 3, 010.

Zhang J, Yang J, Han X, et al (2012). Overexpression of heparanase multiple antigenic peptide 2 is associated with poor prognosis in gastric cancer: Potential for therapy. Oncol 
Lett, 4, 178-82.

Zhang X, Xu S, Tan Q, et al (2013). High expression of heparanase-2 is an independent prognostic parameter for favorable survival in gastric cancer patients. Cancer Epidemiol, 37, 1010-3.

Zheng L, Jiang G, Mei H, et al (2010). Small RNA interferencemediated gene silencing of heparanase abolishes the invasion, metastasis and angiogenesis of gastric cancer cells. BMC Cancer, 10, 33.

Zheng L, Weng M, Qi M, et al (2012). Aberrant expression of intelectin-1 in gastric cancer: its relationship with clinicopathological features and prognosis. J Cancer Res Clin Oncol, 138, 163-72.

Zheng LD, Jiang GS, Pu JR, et al (2009). Stable knockdown of heparanase expression in gastric cancer cells in vitro. World J Gastroenterol, 15, 5442-8.

Cai YG, Fang DC, Yang ShM, et al (2004). The significance of the expression of heparanase mRNA in gastric cancer tissues. J Fourth Military Med University, 909-11.

Chen Bo (2009). Expressions of heparanase and matrix metalloproteinase-9 in gastric cancer and its clinical significance. J Hainan Med College, 852-4

Huang ZHQ, Liu SH, Chen SL, et al (2008). The study of HPA, bFGF and FGFR-2 expressions in gastric cancer tissue. $J$ Modern Chinese Western Integrated Med. 3565, 6-75.

Jiang WH, Zhu XM, Yang ZHP, et al (2011). The expression of heparanase in gastric cancer tissue with its clinical pathological features. Chinese J Gerontol, 3262-3.

$\mathrm{Li}$ Tao, QJ (2012). The expression and significance of integrin alpha 5 beta 1 and heparanase in gastric carcinoma. Guangdong Med. 2974-6.

Liang H, Zhang L, Zhang Xu (2009). The expression and clinical significance of HPA in gastric cancer tissues. Health Vocational Educat, 102-3.

Liu Y, Yang HG, Zuo LF (2007). The expression of heparanase in gastric cancer and DNA content quantitative research by flow cytometry. J Med Res, 40-3.

Liu ZH, An ZhQ, Li EJ, et al (2004). The expressions of heparanase, bFGF fibroblast growth factor expression in gastric cancer with their effects on angiogenesis and tumor progression. Sichuan J Physiological Sci 106-8.

Ma XM, Sun Q, Ren MJ, et al (2007). The expressions of heparanase and NF kappa B in gastric cancer and with the clinical pathological features and vascular formation. $J$ China Histochemistry Cytochemistry, 12-8.

Qi YQ, Si JL, Li WL, et al (2010). The expressions of Syndecan-1 and HPA-1 mRNA in gastric cancer tissue and its relationship with invasion and metastasis of gastric cancer. Chinese $J$ Cancer, 110-5.

Ru GQ, Zhao ZHS, Tang QL, et al (2006). The mRNA expressions of Syndecan-1 and heparanase in gastric cancer and its relationship with metastasis and prognosis of gastric cancer. Chinese J Surg, 1062-4.

Su N, Chen B, Chen JZH, et al (2009). The expression of heparanase in gastric cancer and hepatocellular carcinoma and its clinical significance. J Practical Med, 1207-9.

Sun YS, Ye ZY, a randomised (2005). The expression of heparanase protein in gastric cancer and its clinical significance. Zhejiang Med J, 644, 5-9.

Wang ZhH, Wang YH, Jiang FT, et al (2008). The expressions and significances of heparanase in gastric cancer, paracancerous tissues and normal gastric tissue. Modern Med Oncol, 1953-5.

Zhang L, Zhang X, Yang AJ, et al (2009). The expressions and significances of HPA and HIF-1 in gastric cancer. Shandong J Med Pharmcol, 89-90.

Zheng ZHC, Wang SHB, Xu HM (2004). The expressions of heparanase and bFGF in gastric cancer and its relationship with invasion and metastasis of gastric cancer. Chinese $J$ Clin Oncol, 31, 4-8. 